\title{
Calcinosis cutis congénita tumoral asociada con encefalopatía epiléptica infantil temprana con variante patogénica en el gen FGF12
}

\author{
Eduardo Marín-Hernández ${ }^{*}$, César A. Reyes-Salcedo², Alan Cárdenas-Conejo ${ }^{3}$, \\ Leoncio Peregrino-Bejarano ${ }^{4}$, Araceli Reyes-Cuayahuitt ${ }^{5}$, Darío Rayo-Mares ${ }^{5}$ y Georgina A. Siordia-Reyes ${ }^{6}$ \\ ${ }^{1}$ Servicio de Dermatología Pediátrica, Unidad Médica de Alta Especialidad (UMAE) Hospital de Pediatría, Centro Médico Nacional Siglo XXI, \\ Instituto Mexicano del Seguro Social (IMSS); ${ }^{2}$ Centro Dermatológico Dr. Ladislao de la Pascua; ${ }^{3}$ Servicio de Genética, UMAE Hospital de Pediatría, \\ Centro Médico Nacional Siglo XXI, IMSS; ${ }^{4}$ Servicio de Pediatría, UMAE Hospital de Pediatría, Centro Médico Nacional Siglo XXI, IMSS; ${ }^{5}$ Servicio \\ de Neurología Pediátrica, UMAE Hospital de Pediatría, Centro Médico Nacional Siglo XXI, IMSS; ${ }^{6}$ Servicio de Anatomía Patológica Pediátrica, \\ UMAE Hospital de Pediatría, Centro Médico Nacional Siglo XXI, IMSS. Ciudad de México, México
}

\begin{abstract}
Resumen
Introducción: La calcinosis cutis es el depósito de sales insolubles de calcio en la piel y se clasifica, de acuerdo con su patogénesis, en distrófica, metastásica, idiopática, iatrogénica y calcifilaxis. La calcinosis idiopática se presenta en pacientes sanos y es asintomática; incluye la calcinosis escrotal, la calcinosis nodular de Winer o nódulos calcificados subepidérmicos y la calcinosis tumoral familiar. Esta última es una condición rara que se caracteriza por el depósito de calcio periarticular en pacientes normocalcémicos sin conexión al hueso. Caso clínico: Paciente de sexo masculino de 5 meses de edad, quien al séptimo día de vida fue hospitalizado por ictericia multifactorial, sepsis neonatal tardía y apnea con crisis epilépticas. La evolución fue tórpida, con ingresos hospitalarios por crisis epilépticas de difícil manejo, respuesta parcial a la difenilhidantoína y descontrol electrolítico. Mediante la secuenciación del exoma dirigido se detectó una variante patogénica de sentido equivocado en FGF12 que confirmó el diagnóstico de encefalopatía epiléptica temprana número 47. Además, el paciente presentó dermatosis congénita diseminada a las extremidades inferiores con afección en muslos, asintomática, bilateral y simétrica, constituida por hipopigmentación y fóveas duras a la palpación profunda. La biopsia mostró calcificación distrófica. Conclusiones: Se presenta el caso de un lactante con calcinosis cutis congénita profunda asociada con una variante patogénica en el gen FGF12 y con encefalopatía epiléptica, situación clínica que, a la fecha, no había sido reportada en la literatura.
\end{abstract}

Palabras clave: Calcinosis cutis. Calcinosis cutis tumoral. Epilepsia. Edad infantil temprana.

\section{Tumorous congenital calcinosis cutis associated with early childhood epileptic encephalopathy with a pathogenic FGF12 gene variant}

\begin{abstract}
Background: Calcinosis cutis is the deposit of insoluble calcium salts in the skin. It is classified according to its pathogenesis in dystrophic, metastatic, idiopathic, iatrogenic, and calciphylaxis. Idiopathic calcinosis is asymptomatic, occurs in healthy patients, and includes scrotal calcinosis, Winer's nodular calcinosis or subepidermal calcified nodules, and
\end{abstract}

\section{Correspondencia:}

*Eduardo Marín-Hernández

E-mail: emarinh1973@yahoo.com.mx
Fecha de recepción: 31-01-2020

Fecha de aceptación: 09-05-2020

DOI: 10.24875/BMHIM.20000030
Disponible en internet: 13-11-2020

Bol Med Hosp Infant Mex. 2020;77(6):331-336

www.bmhim.com

1665-1146/C 2020 Hospital Infantil de México Federico Gómez. Publicado por Permanyer. Este es un artículo open access bajo la licencia CC BY-NC-ND (http://creativecommons.org/licenses/by-nc-nd/4.0/). 
familial tumor calcinosis. The latter is a rare condition characterized by periarticular calcium deposition in normocalcemic patients with no bone connection. Case report: The case of a 5-month-old male patient, who on the seventh day of life was hospitalized for multifactorial jaundice, late neonatal sepsis, and apnea with epileptic seizures is described. His evolution was torpid, with hospital admissions due to epileptic seizures that were difficult to manage with partial response to the use of diphenylhydantoin and electrolyte alterations. By means of exome sequencing directed, a pathogenic variant of wrong direction in FGF12 was detected and the diagnosis of early epileptic encephalopathy number 47 was confirmed. Also, the patient showed disseminated congenital dermatosis to lower extremities affecting thighs, asymptomatic, bilateral and symmetrical, constituted by hypopigmentation and fovea hard to deep palpation. The biopsy showed dystrophic calcification. Conclusions: The case of an infant with deep congenital cutis calcinosis associated with a pathogenic variant in the FGF12 gene with epileptic encephalopathy is described. To date, this clinical situation has not been previously reported in the literature.

Key words: Calcinosis cutis. Tumoral calcinosis cutis. Epilepsy. Early childhood.

\section{Introducción}

La calcinosis cutis es el depósito de sales insolubles de calcio en la piel. De acuerdo con su patogénesis, se clasifica en distrófica, metastásica, idiopática, iatrogénica y calcifilaxis. La calcinosis cutis distrófica es la más frecuente y se asocia con enfermedades autoinmunitarias, infecciosas, hereditarias o neoplasias que afectan el aporte de oxígeno en la dermis y la hipodermis, lo que ocasiona necrosis celular. Por su parte, la necrosis ocasiona la liberación de proteínas fijadoras de fosfato y el depósito de hidroxiapatita de calcio en el espacio extracelular, a pesar de que los valores séricos de calcio y fósforo son normales ${ }^{1}$.

La calcifilaxis cutis es el depósito de calcio en la capa media de los vasos de pequeño-mediano calibre de la dermis o del tejido celular subcutáneo. Clínicamente se manifiesta con livedo racemoso o púrpura retiforme.

La calcinosis metastásica se asocia con un incremento de las concentraciones séricas de calcio y fósforo, lo que favorece el depósito de cristales en la piel. La causa más frecuente es la enfermedad renal crónica; otras causas menos frecuentes son el hiperparatiroidismo, la hipervitaminosis $\mathrm{D}$, la sarcoidosis y las neoplasias malignas. Este tipo de calcinosis típicamente se localiza en las articulaciones².

La calcinosis iatrogénica se relaciona con la administración de medicamentos con calcio o fósforo.

La calcinosis idiopática se presenta en pacientes sanos, es asintomática e incluye la calcinosis escrotal, la calcinosis nodular de Winer o nódulos calcificados subepidérmicos y la calcinosis tumoral familiar ${ }^{3}$. La calcinosis tumoral es una condición rara que se caracteriza por el depósito de calcio periarticular en pacientes normocalcémicos sin conexión al hueso ${ }^{4,5}$.

Por otro lado, las encefalopatías epilépticas infantiles tempranas representan un grupo de trastornos del neurodesarrollo. Se identifican por crisis convulsivas tónicas o espasmos de inicio en la infancia con un hallazgo electroencefalográfico específico de patrón brote-supresión caracterizado por picos de alto voltaje que alternan con fases de supresión casi planas ${ }^{6}$. Esta forma de epilepsia grave es una condición genéticamente heterogénea. En la base de datos OMIM (Online Inheritance in Man) se reportan alrededor de 80 diferentes tipos de encefalopatías epilépticas infantiles tempranas, cada uno causado por mutaciones distintas en genes ubicados en diferentes loci. En particular, la variante patogénica en estado heterocigoto en el gen FGF12 localizado en 3q28 causa el cuadro clínico llamado encefalopatía epiléptica infantil temprana número $47^{7,8}$. En este reporte se describe por primera vez la coexistencia de calcinosis cutis profunda congénita tumoral y alguna forma de encefalopatía epiléptica infantil temprana.

\section{Caso clínico}

Se analiza el caso de un paciente de sexo masculino, de 5 meses de edad, producto de primera gestación, normoevolutivo, con parto eutócico a las 37 semanas de gestación. Ingresó a urgencias al séptimo día de vida por ictericia multifactorial, sepsis neonatal tardía y apnea con crisis epilépticas. Durante el internamiento se evidenció neumonía, onfalitis, hernia inguinoescrotal izquierda reducible y $\mathrm{pH}$-metría con datos de reflujo ácido y mixto. Al tercer día de ingreso, el paciente presentó crisis epilépticas (20-30 s), que fueron tratadas con difenilhidantoína, con buena respuesta a las dosis de impregnación y mantenimiento. No se encontraron anomalías en el ultrasonido transfontanelar, y tampoco en la tomografía computarizada de cráneo ni el electroencefalograma. Al mostrar mejoría, se decidió su egreso. 
A los 3 meses, el paciente reingresó al servicio de urgencias por crisis epilépticas descontroladas y evacuaciones amarillentas Bristol V. Se realizó funduplicatura de Nissen. El servicio de neurología evaluó al paciente y reportó monoparesia de extremidad superior izquierda. Se realizó un segundo electroencefalograma que no mostró actividad irritativa cortical de tipo epileptiforme, con lo que se concluyó plexopatía braquial izquierda y se solicitó la evaluación por el servicio de rehabilitación. A la exploración física se observó lateralización cefálica, flacidez de miembro superior izquierdo, aducción y flexión de pie derecho; seguimiento visual deficiente y reflejo de búsqueda nulo; movimientos involuntarios de mandíbula, rigidez de tríceps izquierdo, alteración en la fase I de la deglución y probable disfunción visual. Se realizó un estudio de velocidad de conducción nerviosa y se detectó monoparesia hipotónica de miembro torácico izquierdo por lesión de tronco superior y fascículo medial de plexo braquial. El paciente mostró buena respuesta al tratamiento con difenilhidantoína y egresó para seguimiento en consulta externa.

Al quinto mes de vida, el paciente presentó crisis epilépticas de difícil control asociadas con acidosis metabólica con un valor de brecha aniónica de $22.4 \mathrm{mEq} / \mathrm{l}$ (elevada), por lo que fue necesario un tercer internamiento. Se inició tratamiento con benzodiacepinas, con control parcial. En la interconsulta con la clínica de errores innatos del metabolismo, donde se realizó tamiz metabólico ampliado, no se encontraron alteraciones. Las crisis epilépticas se tornaron de mal control, por lo que el servicio de neurología cambió el esquema anticonvulsivo a levetiracetam. El área de infectología reportó leucocitosis a expensas de linfocitos y radiografía de tórax con patrón reticular bilateral, sin pautar tratamiento. Al cuarto día de hospitalización, el paciente presentó hiperpotasemia, por lo que se iniciaron medidas para contrarrestarla. La resonancia magnética mostró áreas hiperdensas en los tálamos y los núcleos de la base, por lo que se realizó una tomografía computarizada por emisión por fotón único cerebral que mostró áreas de defecto localizadas en la región orbitofrontal y el giro inferior. El paciente egresó por mejoría, pero ingresó nuevamente a los 15 días debido a gastroenteritis aguda y acidosis metabólica, probablemente secundaria a este cuadro (brecha aniónica de $9 \mathrm{mEq} / \mathrm{l}$, normal), así como crisis convulsivas de difícil tratamiento. Fue evaluado por infectología y se indicó tratamiento con antibioticoterapia por cuadro sugestivo de broncoaspiración. El paciente ingresó a la unidad de terapia intensiva pediátrica con hipopotasemia, hipocalcemia e hipermagnesemia. Después de la evaluación

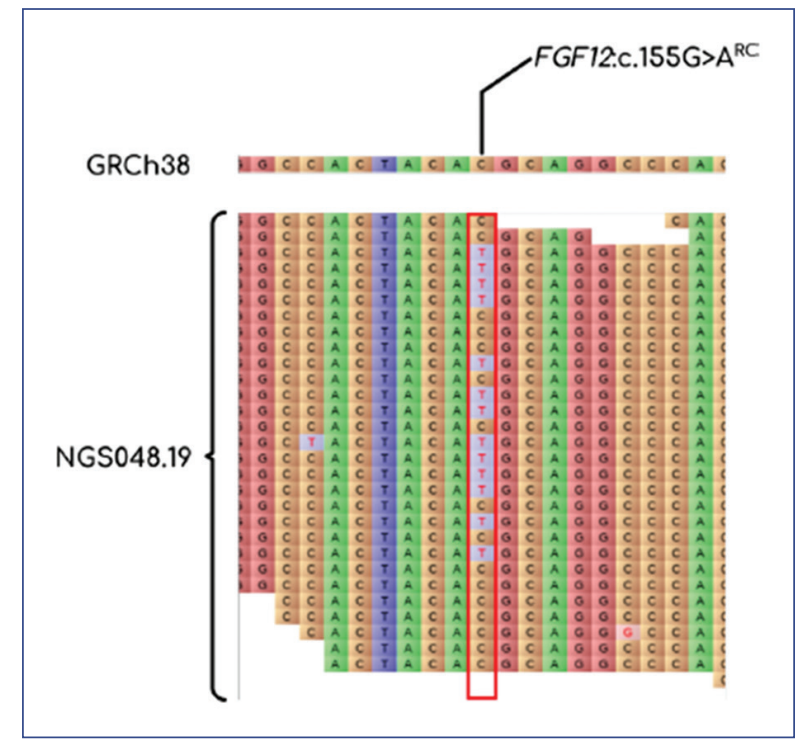

Figura 1. Variante patogénica en el gen FGF12 (señalada en rojo).

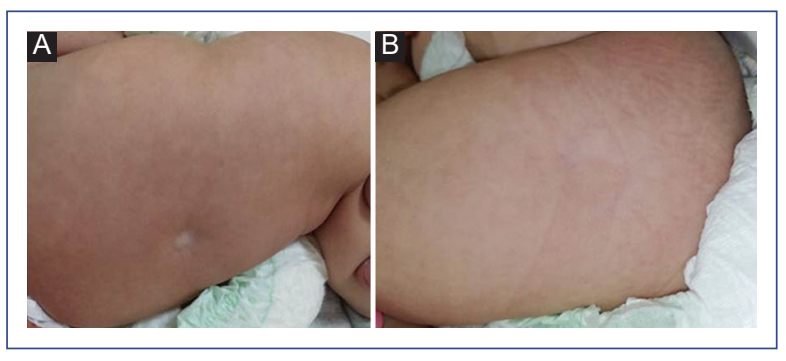

Figura 2. A: Fóvea hipopigmentada en el muslo derecho. B: Neoformación subcutánea en el muslo izquierdo.

por el Departamento de Genética Médica se sugirió la secuenciación del exoma dirigido para el abordaje molecular de un paciente con probable epilepsia de origen monogénico. El resultado de este estudio mostró una variante patogénica de sentido equivocado $c .155 \mathrm{G}>\mathrm{A}$ (p.Arg52His) en FGF12 en estado heterocigoto, que confirmó la encefalopatía epiléptica infantil temprana número 47 (Figura 1). Los padres son aparentemente sanos, por lo que se infirió una mutación de novo.

Se solicitó evaluación por dermatología por dermatosis congénita diseminada a extremidades inferiores con afección en la cara lateral del tercio proximal de los muslos, bilateral y simétrica, constituida por hipopigmentación y fóveas de $2-3 \mathrm{~cm}$ de diámetro que constituyen placas mal definidas, asintomáticas, con dureza a la palpación profunda (Figura 2). Con la impresión diagnóstica de pilomatrixoma versus 


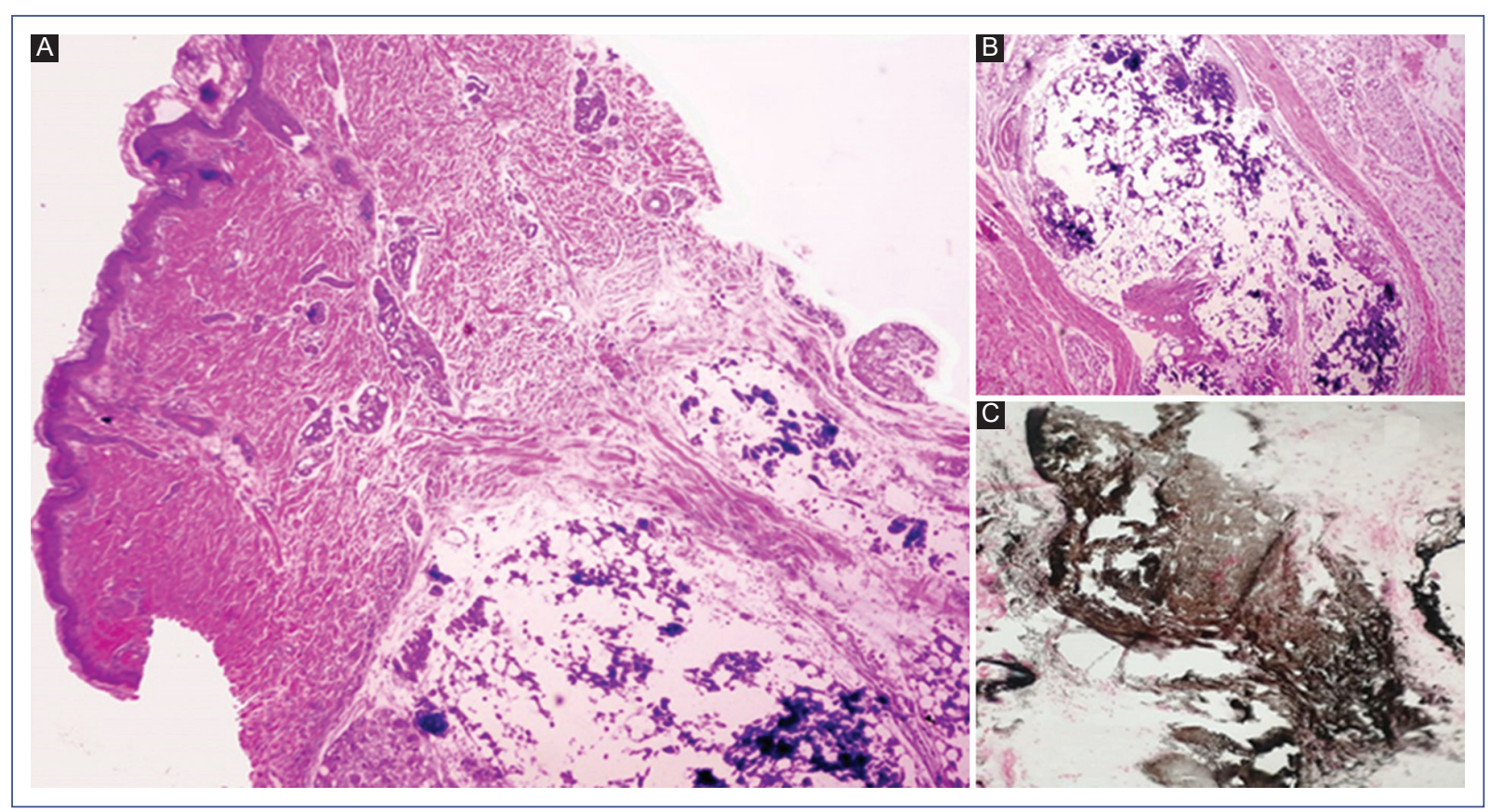

Figura 3. A: Tinción de hematoxilina-eosina (10x). Panorámica en la que se identifica piel delgada con presencia de calcificaciones en el tejido celular subcutáneo. B: Tinción de hematoxilina-eosina (20x). Acercamiento de las calcificaciones. C: Tinción de Von Kossa $(20 x)$ que resalta la presencia de depósito de calcio.

calcinosis cutis, se realizó una biopsia escisional bilateral en la que se observó piel delgada sin alteraciones en la epidermis y la dermis superficial. En la dermis profunda y el tejido celular subcutáneo se identificaron múltiples calcificaciones distróficas rodeadas por fibras de colágena e infiltrado inflamatorio linfohistiocitario leve, sin cambios de necrosis de tejido adiposo, reacción granulomatosa o estructuras radiales dentro de los adipocitos. En un acercamiento se identificó el depósito de calcio en forma granular y sin patrón específico (utilizando la tinción de Von Kossa). Con estos hallazgos se emitió el diagnóstico de calcinosis cutis profunda (Figura 3 ). Se realizó un abordaje elemental del metabolismo mineral fosfocálcico, con los siguientes resultados: calcio sérico total $10.1 \mathrm{mg} / \mathrm{dl}$ (8.5-10.1), calcio iónico 1.12 $\mathrm{mmol} / \mathrm{l}(1.15-1.35)$, fósforo $2.96 \mathrm{mg} / \mathrm{dl}$ (2.5-4.9), vitamina $D 20.8 \mathrm{ng} / \mathrm{ml}$ (30-100), hormona paratiroidea $16.70 \mathrm{pg} / \mathrm{ml}$ (11-67) y calciuria de muestra única 2.9 $\mathrm{mg} / \mathrm{dl}$ (2-17.5). Estos valores se consideraron dentro de los límites normales para la edad, sin implicaciones clínicas.

Actualmente el paciente se encuentra sin crisis epilépticas, en tratamiento con levetiracetam, difenilhidantoína y etosuximida, y en seguimiento con manejo multidisciplinario.

\section{Discusión}

La encefalopatía epiléptica infantil temprana número 47 es una condición neurológica autosómica dominante causada por variantes patogénicas en el gen FGF12, localizado en 3q28 y que codifica al miembro número 1 de la familia de factores homólogos al factor de crecimiento fibroblástico. Esta pequeña proteína citosólica interactúa con el extremo C-terminal de la subunidad alfa del canal de sodio neuronal dependiente de voltaje, modulando su funcionamiento $0^{9,10}$. En líneas celulares se ha demostrado que la mutación identificada en el presente caso genera una ganancia de función, con incremento de la excitabilidad neuronal y la consiguiente actividad epileptiforme ${ }^{11}$. La gravedad del cuadro clínico de esta encefalopatía es variable y la respuesta farmacológica suele ser compleja, aunque se ha informado mejoría al utilizar fenitoína, un bloqueador del canal de sodio dependiente de voltaje.

Por su parte, la calcinosis cutis tumoral consiste en calcificaciones lobuladas bien delimitadas que se localizan en las articulaciones mayores. Histológicamente muestra nódulos amorfos grandes rodeados por una reacción inflamatoria intensa de histiocitos y células gigantes multinucleadas ${ }^{12}$. La condición autosómica recesiva, llamada calcinosis tumoral hiperfosfatémica 
familiar, se caracteriza por el depósito progresivo de cristales de fosfato cálcico en los espacios periarticulares, el tejido blando y, algunas veces, el tejido óseo. Está originada por variantes patogénicas que causan la pérdida de función en alguno de los tres genes relacionados con el metabolismo del fosfato (FGF23, GALNT3 y $K L$ ), por lo que el distintivo bioquímico de la calcinosis tumoral es la hiperfosfatemia secundaria al incremento de la absorción renal del fosfato. Aunque en el paciente no se detectó hiperfosfatemia, la distribución (proximidad a la articulación coxofemoral) y la histología sí fueron compatibles con calcinosis tumoral. Un diagnóstico diferencial a considerar es la necrosis grasa del recién nacido, la cual es una paniculitis transitoria aguda poco frecuente que se desarrolla durante las primeras semanas de vida en productos de término 0 postérmino, y se identifican factores predisponentes maternos y complicaciones perinatales. La patogénesis aún es desconocida, aunque existe la hipótesis de que la hipoxia en el tejido subcutáneo resulta en cristalización y necrosis de adipocitos ${ }^{13}$. Clínicamente, la necrosis grasa del recién nacido se expresa por la presencia de placas o lesiones nodulares subcutáneas induradas, eritematosas, que afectan al tronco, la cara, las nalgas, los muslos o los brazos. Suele ser asintomática, pero podría asociarse con dislipidemia e hipercalcemia, y presentar ocasionalmente depósitos de calcio en el riñón, el corazón, el hígado o la piel ${ }^{14}$. Histológicamente se detecta paniculitis lobulillar en el tejido celular subcutáneo, con adipocitos necróticos, un denso infiltrado inflamatorio con células gigantes multinucleadas y estructuras radiales en el interior de los adipocitos, que corresponden a depósitos de cristales de colesterol. La necrosis grasa del recién nacido muestra un curso benigno y es autorresolutiva ${ }^{13}$.

En el presente caso no se documentó traumatismo directo en las áreas anatómicas involucradas ${ }^{13,14}$. La dermatosis es de presentación bilateral, muy cercana a la articulación coxofemoral y sin evidencia de placa eritematosa previa al desarrollo de la calcinosis. Por otro lado, aun cuando el paciente presentó hipercalcemia, esta fue transitoria, a diferencia de lo que ocurre en la necrosis grasa del recién nacido, en la que se presenta hasta por 6 meses. Tampoco se detectaron los cambios histológicos característicos de la necrosis grasa del recién nacido.

Se desconoce si existe alguna relación biológica entre la ganancia de función de FGF12 como modulador del canal de sodio dependiente de voltaje a nivel neuronal y la presencia de calcinosis cutis — dado que no se ha informado la participación de esta proteína en el control metabólico del calcio y del fósforo a nivel renal-, a diferencia de FGF23, quizá porque pertenece a los factores de crecimiento secretados (FGF1-10 y FGF15-23), mientras que FGF12 pertenece a las formas no secretorias (FGF11-14) de esta familia de factores de crecimiento fibroblástico. Sin embargo, al tratarse de una proteína intracelular, y al no poder ser liberada como ligando a su receptor, FGF12 puede modular el funcionamiento de canales iónicos como los de sodio y calcio, por lo que, además de su implicación en la fisiopatogenia de la encefalopatía epiléptica infantil temprana número 47 , se ha establecido que puede estar relacionada con el desarrollo de taquicardia ventricular idiopática y con la enfermedad de Kashin-Beck, caracterizada por una osteoartropatía endémica deformante con necrosis focales en los condrocitos y crecimiento óseo tubular anormal. Estos datos sugieren que FGF12 podría desempeñar un papel biológico en el crecimiento y el desarrollo óseo ${ }^{7,15}$.

A la fecha no se ha demostrado una relación entre la variante patogénica en estado heterocigoto c.155G $>A$ (p.Arg52His) en FGF12 y el incremento de la probabilidad de desarrollar calcinosis cutis ${ }^{16,17}$. Por ello, el interés del presente estudio fue describir la presentación clinicopatológica sugestiva de una calcinosis cutis tumoral en un paciente con encefalopatía epiléptica infantil temprana número 47 , confirmada por secuenciación de exoma dirigido.

\section{Responsabilidades éticas}

Protección de personas y animales. Los autores declaran que para esta investigación no se han realizado experimentos en seres humanos ni en animales.

Confidencialidad de los datos. Los autores declaran que han seguido los protocolos de su centro de trabajo sobre la publicación de datos de pacientes.

Derecho a la privacidad y consentimiento informado. Los autores han obtenido el consentimiento informado de los pacientes y/o sujetos referidos en el artículo. Este documento obra en poder del autor de correspondencia.

\section{Conflicto de intereses}

Los autores declaran no tener ningún conflicto de intereses.

\section{Financiamiento}

Ninguno. 


\section{Agradecimientos}

Se agradecen el interés y el esfuerzo enfocados en el estudio de la dermatología pediátrica, así como en la elaboración de este reporte.

\section{Bibliografía}

1. Reiter N, El-Shabrawi L, Leinweber B, Berghold A, Aberer E. Calcinosis cutis: part I. Diagnostic pathway. J Am Acad Dermatol. 2011;651:1-12.

2. Jiménez-Gallo D, Ossorio-García L, Linares-Barrios M. Calcinosis cutis and calciphylaxis. Actas Dermosifilogr. 2015;106:785-94.

3. Lai C, Farah R, Mallory S. Congenital calcinosis cutis of the ear. J Am Acad Dermatol. 2003;49:122-4.

4. Amadei F, Petrolati M. Idiopathic tumoral calcinosis associated with congenital malformation of the hand. Ann Chir Main Memb Super. 1998;17:59-62.

5. Karaca L, Polat G, Sade R, Yüce I, Ogul H, Kantarci M. Nontraumatic tumoral calcinosis. Joint Bone Spine. 2017;84:491.

6. Deprez L, Jansen A, De Jonghe P. Genetics of epilepsy syndromes starting in the first year of life. Neurology. 2009;72:273-81.

7. Al-Mehmadi S, Splitt M, Ramesh V, DeBrosse S, Dessoffy K, Xia F, et al FHF1 (FGF12) epileptic encephalopathy. Neurol Genet. 2016;2:1-3.

8. Guella I, Huh L, McKenzie MB, Toyota EB, Bebin EM, ThompsonML, et al De novo FGF12 mutation in 2 patients with neonatal-onset epilepsy. Neurol Genet. 2016:2:1-5
9. Megaloikonomos PD, Mavrogenis AF, Panagopoulos GN, Kontogeorgakos VA. Tumoral calcinosis of the shoulder. Lancet Oncol. 2017;18:e126.

10. Folsom LJ, Imel AE. Hyperphosphatemic familial tumoral calcinosis: genetic models of deficient FGF23 action. Curr Osteoporos Rep. 2015;13:78-87.

11. Siekierska A, Isrie M, Liu Y, Scheldeman C, Vanthillo N, Lagae L, et al. Gain-of-function FHF1 mutation causes early-onset epileptic encephalopathy with cerebellar atrophy. Neurology. 2016;86:2162-70.

12. Li Q, Zhao Y, Wu G, Chen S, Zhou Y, Li S, et al. De novo FGF12 (fibroblast growth factor 12) functional variation is potentially associated with idiopathic ventricular tachycardia. J Am Heart Assoc. 2017;6:1-10.

13. Yori F, Varón S, Valdés B, Acosta MA, De María M, Álvarez M. Necrosis grasa subcutánea del recién nacido: reporte de caso y revisión de la literatura. Arch Pediatr Urug. 2018;89:26-30.

14. Mahé E, Girszyn N, Hadj-Rabia S, Bodemer C, Hamel-Teillac D, De Prost $Y$. Subcutaneous fat necrosis of the newborn: a systemic evaluation of risk factors, clinical manifestations, complications and outcome of 16 children. Br J Dermatol. 2007;156:709-15.

15. Zhang F, Dai L, Lin W, Wang W, Liu X, Zhang J, et al. Exome sequencing identified FGF12 as a novel candidate gene for Kashin-Beck disease. Funct Integr Genomics. 2016;16:13-7.

16. Goldfarb M. Voltage-gated sodium channel-associated proteins and alternative mechanisms of inactivation and block. Cell Mol Life Sci. 2012;69:1067-76.

17. Oda $Y$, Uchiyama $Y$, Motomura A, Fujita A, Azuma $Y$, Harita $Y$, et al. Entire FGF12 duplication by complex chromosomal rearrangements associated with West syndrome. J Hum Genet. 2019;64:1005-4. 